\title{
Optimization for the Online Assessment of Real-time Switch Resistance in Switching Stations
}

\author{
Jiankun Liu \\ Jiangsu Electric Power Company Research Institute \\ Nanjing,China
}

Jing $\mathrm{Bu}$ (corresponding author)

Nanjing University of Science \& Technology

Nanjing,China

bujing30@foxmail.com

\section{Qun Li}

Jiangsu Electric Power Company Research Institute Nanjing,China

\author{
Jing Chen \\ Jiangsu Electric Power Company Research Institute \\ Nanjing,China \\ Lifang Wang \\ Nanjing University of Science \& Technology \\ Nanjing,China \\ Minghui Yin \\ Nanjing University of Science \& Technology \\ Nanjing,China
}

\begin{abstract}
It's important to access the status change of switchgear in switching station for condition maintenance. With regard to the online assessment for the resistance of switches in the switching station with $3 / 2$ connection mode, linear equations can be established on the basis of real-time current measurements. The accuracy of the solution for these linear equations will make a great difference to the whole assessment because of errors in real measurements. To make the calculation for the value of the resistance more accurate, some equations are selected in this paper by comparing matrix condition number. The size of this number reflects the degree of the linear correlation of the equations: the smaller the matrix condition number is, the less influence the variation of coefficient matrix will have on the solution of the equations. Therefore, the condition numbers of all the combinations of coefficient matrixes corresponding to real-time operating conditions should be calculated and the combination with minimum condition number should be selected. In this paper, simulation studies on the current data obtained from $500 \mathrm{kV}$ switchgear are carried out to verify the effectiveness of the proposed method.
\end{abstract}

Keywords - Online Assessment, Switch Resistance, Optimization, Switching Station, Power System

\section{INTRODUCTION}

As the high operational reliability and flexibility, $3 / 2$ connection mode has been the mainstream wiring of the large power plant and high-voltage distribution device of substationat home and abroad ${ }^{[1]}$.The condition monitoring of switching equipment is benefit to arrange maintenance scheduling reasonably, reduce repair cost and achieve condition-based maintenance of break. So, it's important to maintain the normal operation of the transformer substation ${ }^{[2]}$

Currently, the research about online monitoring of the switchgear is primarily interested in the electrical endurance and mechanical characteristics of high-voltage breaker contact. There are a lot of new ways like acoustic diagnosis and non-invasive diagnostic ${ }^{[3][4]}$. In China, some instruments have been developed the instrument to monitor the operation state of high-voltage breaker, but them need to be made better ${ }^{[5]}$. To assess the resistance of switch in switching station with $3 / 2$ connection mode, a method for real-time online assessment on the basis of current measurement is proposed. Based on Kirchhoff's current and voltage laws as well as Ohm's Law, the real-time current online is used to establish linear homogeneous equations. While, the equation we established maybe morbid due to the relativity between real-time currents, then it will cause errors. To morbid equations, we have obtained some solutions like conditions pre-better method; iterative corrections method; projection method; rigid constant differential method and genetic algorithm; etc. However, the vast majority of algorithms are impossible to satisfy both validity and simplicity. Also, to the different morbid degrees and scales they are difficult to meet the precision that we need ${ }^{[6]}$.

As considering the error of equations comes from the error of current and morbid equation caused by the relativity between real-time currents, we select equations in this article is by comparing the matrix condition number ${ }^{[7]}$. Based on many conditions that can be measured, we have multiple combinations of the coefficient matrix .The only thing need to do is to traverse all the combinations and select the combination with minimum condition number to calculate. Then, we can make the evaluation parameters more accurate and provide a basis for the choice of current condition of the equations that established.

To verify the effectiveness, this article uses the actual current condition of substation one day to calculate the resistance. 


\section{THE RELATIONSHIP BETWEEN CONDITION NUMBER AND RELATIVE ERROR ON THE SOLUTION OF THE EQUATIONS.}

In mathematics, the condition number is defined as: for the matrix $A$, its condition number is equal to the product of the norm of matrix $A$ and the norm of inverse matrix $\quad A, \quad$ i.e., $\quad \operatorname{cond}(A)=\|A\| *\left\|A^{-1}\right\|$.The condition number can react the error or uncertain sensitivity in $\mathrm{A}$ and $\mathrm{b}$ which are in $A \cdot x=b$ [8].

There is a $N$-Order linear equation: $A \cdot x=b$.If there is a small disturbance $\delta A$ and $\delta b$ in the coefficient matrix, the equation under this disturbance is:

$$
(A+\delta A)(x+\delta x)=b+\delta b
$$

So that:

$$
\frac{\|\delta x\|}{\|x\|} \leq \frac{\operatorname{cond}(A)}{1-\operatorname{cond}(A) \frac{\|\delta A\|}{\|A\|}}\left(\frac{\|\delta A\|}{\|A\|}+\frac{\|\delta b\|}{\|b\|}\right)
$$

As the formula 2 shows, the small disturbance in A and $\mathrm{b}$ will cause grater error when the condition number (Cond $(A))$ is bigger. So the condition number (Cond (A)) describes the behavior of the equation $(A \cdot x=b)$. If the small changes in $\mathrm{A}$ and $\mathrm{b}$ cause the huge changes in the solution of the equation. We call this equation the morbid equation group and the coefficient matrix $\mathrm{A}$ is morbid matrix. While equation is well-conditioned linear equation when the change is little. If the condition number is more than 100 , we can judge the equation $(A \cdot x=b)$ is morbid. 'Morbid and well-condition' is a relative concept.

In physics, the solution of the morbid equation is the intersection of two approximate parallel lines. Subtle changes will lead to the new intersection far from the old one. So, the solution is influenced by disturbance greatly.

For example, the precision of the equation $\left(\begin{array}{cc}2 & 6 \\ 2 & 6.0001\end{array}\right)\left(\begin{array}{l}x_{1} \\ x_{2}\end{array}\right)=\left(\begin{array}{c}8 \\ 8.0001\end{array}\right)$ is $x=(1,1)^{T}$. If a small change in $A$ and $b$, such as $\left(\begin{array}{cc}2 & 6 \\ 2 & 5.9999\end{array}\right)\left(\begin{array}{l}x_{1} \\ x_{2}\end{array}\right)=\left(\begin{array}{c}8 \\ 8.0002\end{array}\right)$, the solution of this equation becomes $x=(10,-2)^{T}$. The solution changes a lot because of the subtle disturbance. This is the morbid phenomenon ${ }^{[9]}$.

\section{OPTIMAL SELECTION OF THE COEFFICIENT MATRIX.}

\section{A. The establishment of linear equations.}

Supposing there are $m$ three-circuit-breaker branches and $n$ two-circuit-breaker branches in a substation with the $3 / 2$ connection mode. The diagram of substation with the $3 / 2$ connection mode is showed in Fig .1. The measured current of each switch in a moment is: $i_{1} i_{2} i_{3} \mathrm{~L} i_{3 m+2 n-1} i_{3 m+2 n}$, the resistance of each switch is: $R_{1} R_{2} R_{3} \mathrm{~L} R_{3 m+2 n-1} R_{3 m+2 n}$.

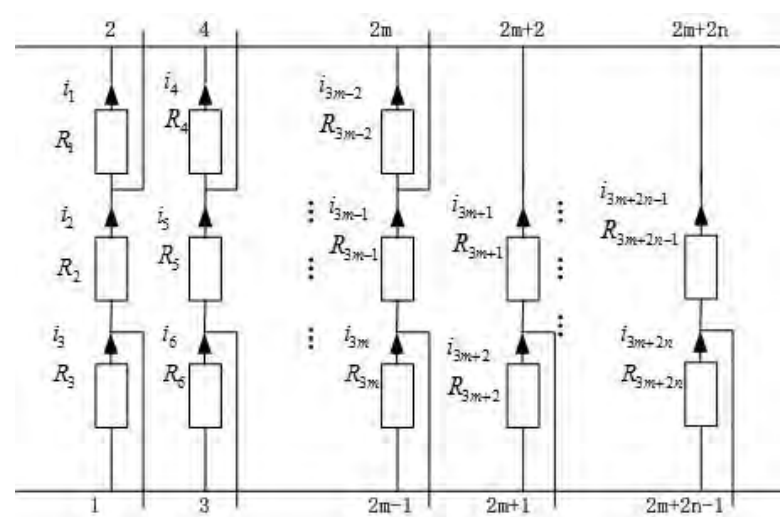

Figure 1. Diagram of 3/2 wiring transformer substation

Assuming $R_{1}$ is reference resistance, i.e., $R_{1}=1$, others expressed by the ratio of $R_{1}$. Based on Kirchhoff's voltage law, we can get linear equation after mathematical manipulation:

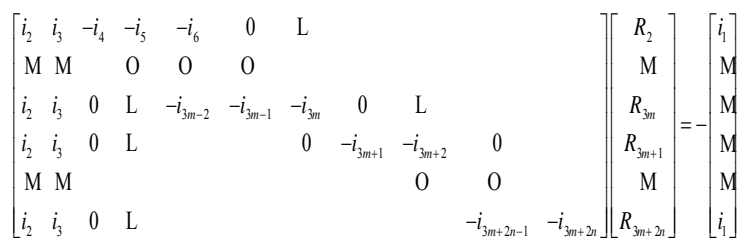

Supposing the number of current conditions is $N$, the number of unknowns is $3 m+2 n-1$ and the number of equations is $N^{*}(m+\mathrm{n}-1)$. We should ensure that the number of equations is no less than the number of unknowns.

\section{B. Analyzation of error sources about the solution of linear equations}

1) Measurement error of coefficient matrix The improved algorithm can measure the current of each branch at the same time in a substation with $3 / 2$ connection mode. So, the reliability of this algorithm is influenced by the precision of current transform.

Currently, according to the different function of the secondary winding, current transform usually can be divided into two categories: measuring current transformer and protective current transformer.

The first kind is the measuring current transformer. 
To ensure the right measure, the output of this current transformer must meet the required accuracy class under normal operating conditions.

According to the standard, the following table shows the limitation of current error and phase error in rated frequency, when accuracy class of measuring current transformer is 0.2 or 0.5 and secondary load is between $25 \%$ and $100 \%{ }^{[10]}$.

TABLE I. THE LIMITATION OF ERROR TO MEASURING CURRENT TRANSFORMER

\begin{tabular}{|c|c|c|c|c|c|c|c|c|c|c|c|c|}
\hline \multirow{3}{*}{$\begin{array}{l}\text { Accuracy } \\
\text { class }\end{array}$} & \multirow{2}{*}{\multicolumn{4}{|c|}{$\begin{array}{c}\text { Current error } \pm \% \\
\text { (in following rated current } \% \text { ) }\end{array}$}} & \multicolumn{8}{|c|}{ Phase error (in following rated current $\%$ ) } \\
\hline & & & & & \multicolumn{4}{|c|}{$\pm(')$} & \multicolumn{4}{|c|}{$\pm \mathrm{rad}$} \\
\hline & 5 & 20 & 100 & 120 & 5 & 20 & 100 & 120 & 5 & 20 & 100 & 120 \\
\hline 0.2 & 0.75 & 0.35 & 0.2 & 0.2 & 30 & 15 & 10 & 10 & 0.9 & 0.45 & 0.3 & 0.3 \\
\hline 0.5 & 1.5 & 0.75 & 0.5 & 0.5 & 90 & 45 & 30 & 30 & 2.7 & 1.35 & 0.9 & 0.9 \\
\hline
\end{tabular}

The second kind is the protective current transformer[11].One of the basic requirements is to ensure the error keep within limits under a certain amount of overcurrent.The accuracy class of protective current transformer includes 5Pand 10P in the national standard GB1208.The error limits under rated frequency and rated load are shown in following table:

TABLE II. THE LIMITATION OF ERROR TO PROTECTIVE CURRENT TRANSFORMER

\begin{tabular}{|c|c|c|c|}
\hline $\begin{array}{c}\text { the class of } \\
\text { protective current } \\
\text { transformer }\end{array}$ & Current error $\pm(\%)$ & Phase error $\pm\left({ }^{\prime}\right) / \pm$ rad & $\begin{array}{c}\text { The composite error of } \\
\text { primary current of the } \\
\text { ratedaccuratelimitation }(\%)\end{array}$ \\
\cline { 2 - 4 } & 1 & $60 / 1.8$ & 5 \\
\hline 5P & 3 & - & 10 \\
\hline $10 \mathrm{P}$ & $1 \%$ & \\
\hline
\end{tabular}

From the data in tables above, we can see that the measurement error cannot be avoided to the current transformer.

\section{2) The Correlation of Coefficient Matrix}

In Fig .1,assuming the current direction of the entire current outlet is upward. We can get the sum of the current which flow in and out the switching station is zero based on Kirchhoff's current law. So that:

(4)

$\left(i_{2}-i_{1}\right)+\left(i_{5}-i_{4}\right)+\Lambda+\left(i_{3 m-1}-i_{3 m-2}\right)=\left(i_{2}-i_{3}\right)+\left(i_{5}-i_{6}\right)+\Lambda+\left(i_{3 m+2 n-1}-i_{3 m+2 n}\right)$

As the formula 4 shows, there is a coupling relationship among $i_{1}, i_{2} \Lambda i_{3 m+2 n}$.And the coefficient matrix in formula 3 is made of different conditions of them. So there is a certain correlation among the row vectors of the coefficient matrix ${ }^{[12]}$, although the current sampling values come from different conditions. And the linear correlation ofrow vector of the matrix is closely related with the conditions. The relevance of coefficient matrix will lead to larger condition number, and made the equation morbid. Due to the error of

$\operatorname{Min}\left(\operatorname{cond}\left(\mathrm{A}_{\left(1: C_{N \cdot(m+n-1)}^{3 m+2 n-1}\right)}\right)\right)$ kinds of combinations should be selected to correlate.

From formula 2, we know that the accuracy of solutions was determined by the size of Cond $(A)$ when the value of $\delta A$ and $\delta b$ was known.

The selection principle of coefficient matrix is: The smaller the condition number of the coefficient matrix is, the higher the accuracy of the solution will be. transformer measurement, subtle errors will lead to the large error in the solution if condition number becomes lager

According to the relationship between the coefficient matrix condition and the precision of the solution in the second chapter, the precision of the solution is influenced by the correlation of the row vector of coefficient matrix which is shown in formula4.

\section{The Optimal Selection of Current Condition to Establish Equations}

A variety of current conditions can be measured to establish the linear equations for getting the solution of switchgear.When the number of equations is more than the number of unknowns, we need to select some equations with the lowest correlation from all to calculation. It's benefit to improve the precision ${ }^{[13]}$.

To formula 3 , if the number of measuring condition is $\mathrm{N}$, we need to calculate the conditions including $C_{N \cdot(m+n-1)}^{3 m+2 n-1}$ kinds of combinations. Then,

Therefore, when choosing equations, the only thing need to do is to traverse all the combinations and select the combination with minimum condition number. Then calculate the resistance of switchgear based on Gaussian elimination with this coefficient matrix.

\section{SimULATION ANALYSIS}

To verify the effectiveness of the method, we can do a simulation by using the current data from a $500 \mathrm{kV}$ 
switchgear of $3 / 2$ connection mode. Fig .2 is the wiring diagram of the switching station. Table 3 shows the selected current data according to the operation in a quarter of this switching station.

TABLEIII. THE CURRENT DATA FLOWING THROUGH THE SWITCH AT DIFFERENT TIMES

\begin{tabular}{cccccccccccc}
\hline$I(A)$ & $i_{1}$ & $i_{2}$ & $i_{3}$ & $i_{4}$ & $i_{5}$ & $i_{6}$ & $i_{7}$ & $i_{8}$ & $i_{9}$ & $i_{10}$ & $i_{11}$ \\
\hline 1 & 273.56 & -626.79 & -76.86 & 183.93 & -616.07 & 83.93 & 132.53 & -768.78 & 32.44 & -589.91 & -39.62 \\
2 & 173.87 & -526.74 & 73.62 & 133.27 & -366.86 & -16.74 & 302.54 & -597.46 & -197.46 & -609.39 & 140.61 \\
3 & 121.35 & -379.18 & -28.83 & 325.12 & -474.88 & -74.88 & -16.97 & -417.28 & 83.06 & -429.40 & 20.60 \\
4 & 193.82 & -706.55 & 193.74 & 4.82 & -395.31 & 104.89 & 334.47 & -615.53 & -315.53 & -533.17 & 17.04 \\
5 & -194.04 & -493.99 & 306.60 & 397.01 & -502.99 & -152.99 & 328.64 & -621.36 & -221.36 & -533.31 & 68.15 \\
6 & 208.81 & -592.20 & 8.60 & 250.68 & -600.22 & 50.49 & 177.00 & -623.00 & -123.00 & -636.07 & 63.93 \\
7 & 152.73 & -598.34 & 52.60 & 67.53 & -532.47 & 167.53 & 57.81 & -592.19 & 7.81 & -277.94 & -228.25 \\
8 & 205.03 & -544.97 & -44.97 & -27.94 & -527.94 & 272.06 & 210.29 & -689.71 & -89.71 & -387.37 & -137.37 \\
\hline
\end{tabular}

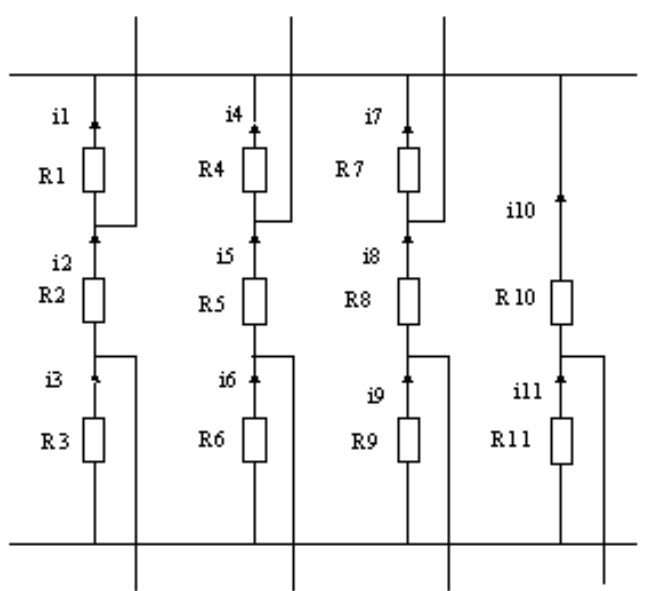

Figure 2. Circuit diagram of a $500 \mathrm{kV}$ switching station
We need to select all the combinations of the equations, and get the condition number of all. Then compare the coefficient matrix whose condition number is 88.66 with the one of 370.52 . Finally, we pick out two kinds of combination coefficientsto establish equations. After that, the resistance value should be calculated.

When the condition number is small, the accuracy is higher than the situation that condition number is larger. The error is under $20 \%$, when condition number is 88.66 , and the error is over $20 \%$, when condition number is 370.52 . So, the rationality of this optimal algorithm is verified by this example.

TABLEVI. COMPARES OF THE SWITCH RESISTANCE (CONDITION NUMBER IS 88.66)

\begin{tabular}{cccccccccccc}
\hline & $R_{1}$ & $R_{2}$ & $R_{3}$ & $R_{4}$ & $R_{5}$ & $R_{6}$ & $R_{7}$ & $R_{8}$ & $R_{9}$ & $R_{10}$ & $R_{11}$ \\
\hline$V_{\text {real }}$ & 1.00 & 1.50 & 1.30 & 1.80 & 2.00 & 1.60 & 1.50 & 1.30 & 1.00 & 1.20 & 1.50 \\
Vcal & 1.00 & 1.50 & 1.30 & 1.80 & 2.00 & 1.60 & 1.52 & 1.30 & 1.02 & 1.20 & 1.50 \\
Err $(\%)$ & 0.00 & 0.00 & 0.00 & 0.00 & 0.00 & 0.00 & 1.33 & 0.00 & 2.00 & 0.00 & 0.00 \\
\hline
\end{tabular}

TABLEV. COMPARES OF THE SWITCH RESISTANCE(CONDITION NUMBER IS 370.52)

\begin{tabular}{cccccccccccc}
\hline & $R_{1}$ & $R_{2}$ & $R_{3}$ & $R_{4}$ & $R_{5}$ & $R_{6}$ & $R_{7}$ & $R_{8}$ & $R_{9}$ & $R_{10}$ & $R_{11}$ \\
\hline$V_{\text {real }}$ & 1.00 & 1.50 & 1.30 & 1.80 & 2.00 & 1.60 & 1.50 & 1.30 & 1.00 & 1.20 & 1.50 \\
Vcal & 1.00 & 1.47 & 1.29 & 1.81 & 1.97 & 1.61 & 1.47 & 1.27 & 0.97 & 1.17 & 1.46 \\
Err $(\%)$ & 0.00 & 2.00 & 0.76 & 0.56 & 1.50 & 0.63 & 2.00 & 2.31 & 3.00 & 2.50 & 2.67 \\
\hline
\end{tabular}

\section{CONCLUSIONS}

In this paper, the optimization method that uses the condition number of coefficient matrix as a basis to select coefficient matrix is used to establish the linear equations to get the switch resistance of switch station.
This method not only can reduce the correlation between the row vectors of coefficient matrix, but also can reduce the adverse effects caused by the error of current measure.This method traverses all the combinations of coefficient matrixes and select the combination with minimum condition number to 
establish equations to solve. After test and verify, the method we used in this paper can improve the precision of the solution and will improve the accuracy of the online assessment for switch status.

\section{REFERENCES}

[1] Sun Ruizhang. Basis of Electrical Engineering[M]. China Water \& Power Press, 1995(in Chinese).

[2]Wang Junxia. The condition assessment method for HV circuit breakers based on FAHP[D]. Sichuan: Xihua University, 2013(in Chinese).

[3] Goto K, Sakakibara T, Kamata I, et al. On-line monitoring and diagnostics of gas circuit breakers[J]. Power Delivery, IEEE Transactions on, 1989, 4(1): 375-381.

[4] Hoidalen H K, Runde M. Continuous monitoring of circuit breakers using vibration analysis[J]. Power Delivery, IEEE Transactions on, 2005, 20(4): 2458-2465.

[5] Huang Zhongqun. The condition monitoring of SF6 circuit breaker[J]. Fujian Power and Electrical Engineering, 2002.1: 59 -60 .
[6] Lin Shengliang. Studying the algorithm for solving I11-conditioned linear system of equations[D]. Hangzhou: Zhejiang University, 2005(in Chinese).

[7] Wang Caihua, Zheng Shangkun. Discussion of some problems about condition number and perturbation theory of linear equations[J]. College Mathematics, 2013, 29(4): 70-74.

[8] Wang Zhenglin, Liu Ming, Chen Liangui. Matlab Application[M] Beijing: Publishing House of Electronics Industry, 2013.

[9] Xie Xiudong, Lei Jigang, Zhan Guizhi, et al. Theory and Methods of Matrixes[M]. Beijing: Science Press, 2012(in Chinese).

[10] Li Lan. The interpretation and analysis of JJG313-2010 "measuring current transformer". Science \& Technology Information, 2012, 23: 243-244.

[11] Shi RongZhang, Tanwei. The selection and calibration of current transformers[J]. Electrotechnical Application, 2011,30(11): 64-66.

[12] Huang Junqing. Practical Method for Modeling Static or Dynamic Mathematical Model[M]. Beijing: China Machine Press, 1988.

[13] Wang Xiaofei. Condition numbers of two kinds of special matrices[D]. Taiyuan: Taiyuan University of technology, 2013(in Chinese). 